\title{
A Classification of Spherical Curves Based on Gauss Diagrams
}

\author{
Guy Valette ${ }^{1}$
}

Received: 28 August 2015 / Revised: 4 May 2016 / Accepted: 23 June 2016 /

Published online: 11 July 2016

(C) Institute for Mathematical Sciences (IMS), Stony Brook University, NY 2016

\begin{abstract}
We consider generic smooth closed curves on the sphere $S^{2}$. These curves (oriented or not) are classified relatively to the group $\operatorname{Diff}\left(S^{2}\right)$ or its subgroup $\operatorname{Diff}^{+}\left(S^{2}\right)$ ), with the Gauss diagrams as main tool. V. I. Arnold determined the numbers of orbits of curves with $n$ double points when $n<6$. This paper explains how a preliminary classification of the Gauss diagrams of order 5, 6 and 7 allows to draw up the list of the realizable chord diagrams of these orders. For each such diagram $\Gamma$ and for each Arnold symmetry type $T$, we determine the number of orbits of spherical curves of type $T$ realizing $\Gamma$. As a consequence, we obtain the total numbers of curves (oriented or not) with 6 or 7 double points on the sphere (oriented or not) and also the number of curves with special properties (e.g. having no simple loop).
\end{abstract}

Keywords Sphere - Smooth closed curve - Double point · Diffeomorphism . Enumeration

\section{Introduction}

In this paper, a spherical curve will be a generic smooth closed curve on $S^{2}$, more explicitly the image of the unit circle under an immersion $f: S^{1} \rightarrow S^{2}$, the multiple points of which are double points $f(a)=f(b)(a \neq b)$ with distinct tangent directions.

Electronic supplementary material The online version of this article (doi:10.1007/s40598-016-0049-3) contains supplementary material, which is available to authorized users.

$凶$ Guy Valette

guy.valette@vub.ac.be

1 Department of Mathematics, Vrije Universiteit Brussel, Pleinlaan 2, B1050 Brussels, Belgium 
We consider not only spherical curves (often denoted by $C$ ) but also oriented spherical curves (denoted by $C^{+}$and $C^{-}$). Also the sphere may be oriented; this allows to define orientation-preserving $C^{\infty}$-diffeomorphisms of $S^{2}$; the group of such diffeomorphisms will be denoted by $\operatorname{Diff}^{+}\left(S^{2}\right)$. When $G$ is a group acting on a set $X$, one says that two objects $U, V$ are $G$-equivalent if there is an element of $G$ mapping $U$ onto $V$. In particular, it makes sense to consider $\operatorname{Diff}\left(S^{2}\right)$-equivalent or $\operatorname{Diff}^{+}\left(S^{2}\right)$-equivalent curves or oriented curves.

We take an interest in the classifications of spherical curves. To begin with, we consider curves without double points. It is known that two oriented spherical curves without double point are $\operatorname{Diff}^{+}\left(S^{2}\right)$-equivalent and, a fortiori, that two spherical curves without double point are $\operatorname{Diff}^{+}\left(S^{2}\right)$-equivalent. An analogous property is true for oriented curves with just one double point, but not for oriented curves with more than one double point: the number of $\operatorname{Diff}^{+}\left(S^{2}\right)$-orbits is then larger than 1. Results of V.I. (Arnold 1994, p. 27) over the numbers of orbits of spherical curves with at most five double points are summarized in Table 1 .

The last row contains the numbers of orbits of unoriented spherical curves with $n$ double points for $n$ in $\{0,1, \ldots, 5\}$, relatively to the $\operatorname{Diff}\left(S^{2}\right)$-equivalence; the other rows contain the numbers of orbits when $S^{2}$ or $S^{1}$ are oriented.

The method used by Arnold (1996) for computing the numbers of orbits of spherical curves becomes very long when the number $n$ of double points is larger than 5 ; this prompted us to look for another way to compute them. The method presented in this paper is essentially based on a structured classification of combinatorial objects, the Gauss diagrams, which summarize the intrinsic geometry of the curves (one forgets that the curves are contained in a sphere). With this method, we first verified the correctness of the values in Table 1 , and afterwards we found the values for $n=6$ and $n=7$, given in Table 2 .

With Arnold we shall say that a curve is irreducible if it cannot be disconnected by removing one double point. As they are building blocks for all other curves, it is

Table 1 Numbers of orbits of spherical curves found by Arnold

Table 2 Numbers of orbits of spherical curves with 6 or 7 double points

\begin{tabular}{llllllr}
\hline Oriented object(s) & $n=0$ & 1 & 2 & 3 & 4 & 5 \\
\hline$S^{2}$, curves & 1 & 1 & 3 & 9 & 37 & 182 \\
$S^{2}$ & 1 & 1 & 2 & 6 & 21 & 99 \\
curves & 1 & 1 & 2 & 6 & 21 & 97 \\
none & 1 & 1 & 2 & 6 & 19 & 76 \\
\hline
\end{tabular}

\begin{tabular}{lrl}
\hline Oriented object(s) & $n=6$ & 7 \\
\hline$S^{2}$, curves & 1143 & 7553 \\
$S^{2}$ & 588 & 3829 \\
Curves & 579 & 3812 \\
None & 376 & 2194 \\
\hline
\end{tabular}



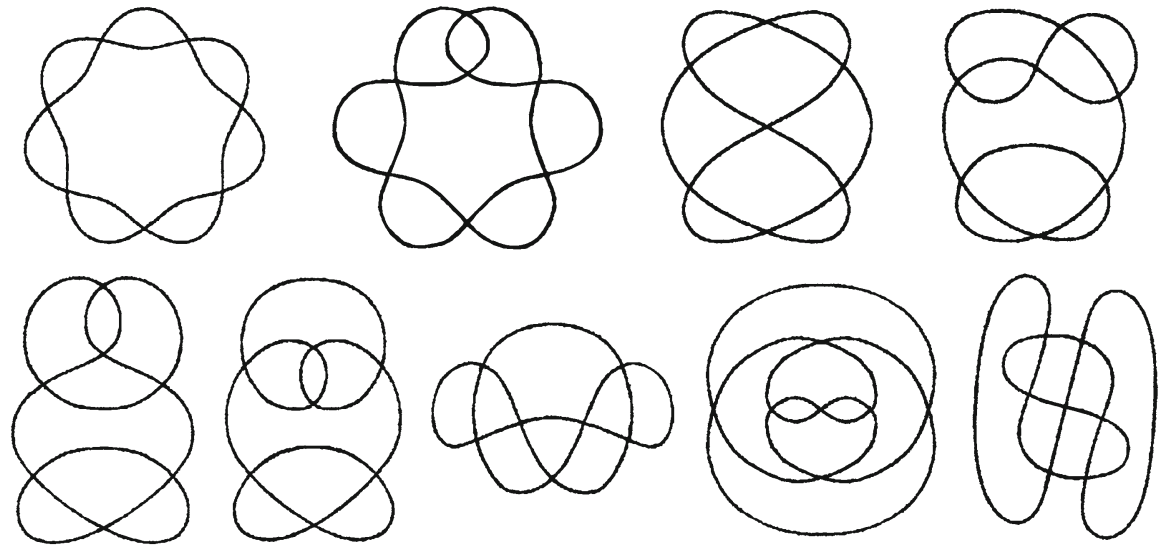

Fig. 1 Arnold's irreducible curves with 7 double points
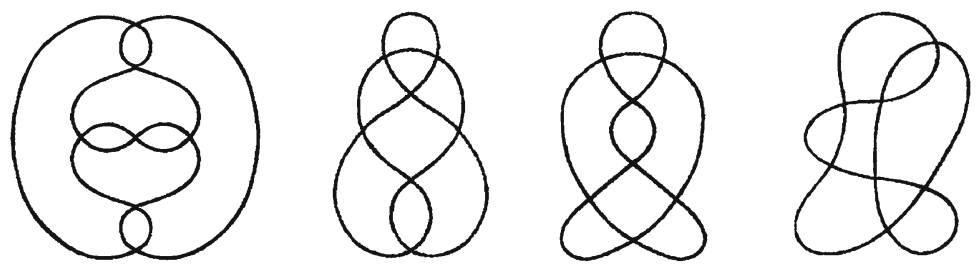

Fig. 2 Supplementary irreducible curves with 7 double points
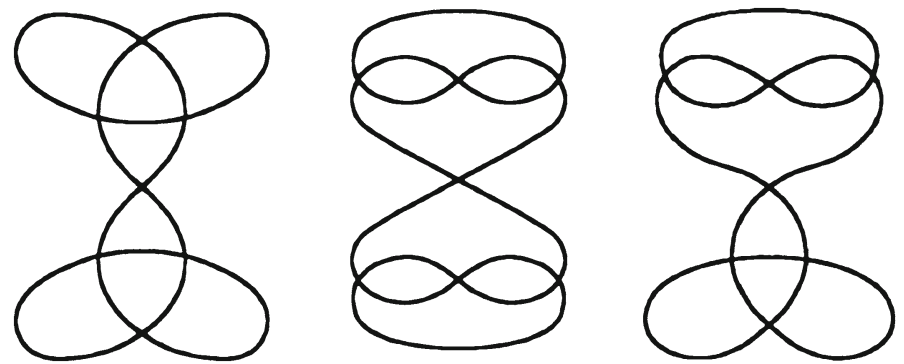

Fig. 3 Reducible curves with 7 double points, without any simple loop

important to know them concretely. Arnold (1996) described 9 irreducible curves with 7 double points, represented in Fig. 1.

To get a complete list of representatives of all irreducible curves with 7 double points, one must add four elements, represented in Fig. 2.

No part of an irreducible curve is a simple loop (closed curve which is $\operatorname{Diff}\left(S^{2}\right)$ equivalent with the right part of the symbol infinity). If we are looking for representatives of all spherical curves with 7 double points and without any simple loop, we must add to the curves of Figs. 1 and 2 the curves of Fig. 3.

These results and many other which will appear further are consequences of a previous determination of all the Gauss diagrams of order 6 and 7. 


\section{Families of Gauss Diagrams}

Let $\gamma: S^{1} \rightarrow S^{2}$ be an immersion whose image is a curve $C$ with $n$ double points and let $D$ be the closed disk bounded by $S^{1}$; for every pair $a, b$ of points of $S^{1}$ which determine a double point of $C$, one may draw a line segment joining $a$ and $b$; the figure consisting of the disk $D$ and these $n$ line segments is the Gauss diagram $\Gamma(\gamma)$. The $n$ segments are the chords and their $2 n$ endpoints are the nodes of $\Gamma(\gamma)$.

As we are interested in the curves $\gamma\left(S^{1}\right)$ and not of the immersions $\gamma$, it is not a restriction to suppose that the nodes of every Gauss diagram are regularly placed on $S^{1}$ (they are the vertices of a regular $2 n$-gon).

As a matter of fact, the Gauss diagram of $\gamma$ is a graphical representation of a combinatorial object related with the curve $\gamma\left(S^{1}\right)$ which is defined below when $n$ is an integer larger than 1 (there are minor changes when $n=0$ or 1 ).

Definition A chord diagram of order $n$ is a set $\mathcal{A}$ of $2 n$ elements, the nodes, provided with two families $\mathcal{E}$ and $\mathcal{C}$ :

(a) The elements of $\mathcal{E}$, called edges, are pairs of nodes such that $(\mathcal{A}, \mathcal{E})$ is a circuit graph (if the chord diagram comes from $\Gamma(\gamma)$, the edges come from endpoints of chords which are neighbours on $S^{1}$ );

(b) The elements of $\mathcal{C}$, called chords, are disjoint pairs of nodes whose union is $\mathcal{A}$ (if the chord diagram comes from $\Gamma(\gamma)$, the elements of $\mathcal{C}$ come from the chords of $\Gamma(\gamma))$.

As $(\mathcal{A}, \mathcal{E})$ is a connected graph, two nodes have a distance: the smallest number of edges needed to join the nodes. In a chord diagram, the step of a chord $a b$ is the distance between $a$ and $b$. The following property of $\Gamma(\gamma)$ was observed by Gauss (1900): along the arcs of $S^{1}$ limited by endpoints of every chord, the number of nodes is always even; this suggests a

Definition A Gauss diagram of order $\mathrm{n}$ is a chord diagram of order $n$ satisfying the parity condition: the step of every chord is odd.

For example, the first figure below represents a Gauss diagram, but the second one does not:
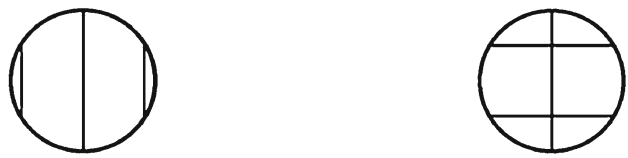

Isomorphic Gauss diagrams will be identified; so there is only one Gauss diagram of order 0 , one of order 1 and one of order 2, but three of order 3 .

Remark A Gauss diagram of order $n$ is not necessarily related with a generic spherical curve having $n$ double points; this Gauss realizability problem was solved on different ways in papers of Francis (1969), Marx (1969), Lovasz and Marx (1976), Rosenstiehl and Read (1977), Dowker and Thistlethwaite (1983), Chaves and Weber (1994). 
For example, the following Gauss diagrams are not realizable:
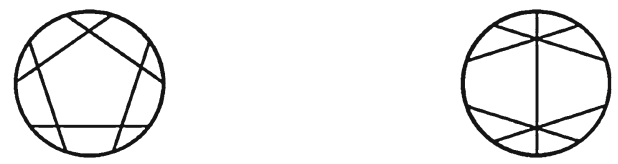

Definition The distinct chords $a b$ and $c d$ of a Gauss diagram $\Gamma$ are interlaced if $a, c, b, d$ are in cyclic order along the circuit graph of $\Gamma$; with Rosenstiehl and Read, we define the interlacing graph of $\Gamma$ as follows: its nodes are the chords of $\Gamma$ and its edges are the pairs of interlaced chords.

New Look of Diagrams: if one represents chords of a Gauss diagram by chords of a circle, the chords the step of which is small w.r.t. the order of a Gauss diagram are not very visible; for this reason and for aesthetic care, we replace the line segments by circular arcs in the drawing of chords. For example, the diagram showed below on the left has the new look drawn on the right:
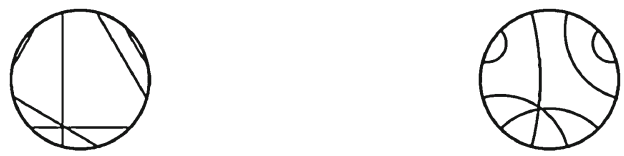

We are now in a position to define the families of Gauss diagrams; we begin with the notion of fatherhood for diagrams.

Definition If the Gauss diagram $\Gamma=(\mathcal{A}, \mathcal{E}, \mathcal{C})$ has at least one chord the step of which is 1 , the father-diagram of $\Gamma$ is the diagram $\Gamma^{\prime}=\left(\mathcal{A}^{\prime}, \mathcal{E}^{\prime}, \mathcal{C}^{\prime}\right)$ obtained as follows:

(a) $\mathcal{C}^{\prime}$ is the set of the chords of $\Gamma$ whose step is larger than 1 ;

(b) $\left(\mathcal{A}^{\prime}, \mathcal{E}^{\prime}\right)$ is the circuit graph obtained by deleting the nodes which are endpoints of chords of $\Gamma$ with step 1, and respecting the cyclic order of the preserved nodes (it is the empty graph if $\mathcal{C}^{\prime}$ is empty).

For example, the father-diagram of the Gauss diagram of order 6 represented below by the first drawing is the Gauss diagram of order 4 represented by the second one, which is equivalent to the third one:
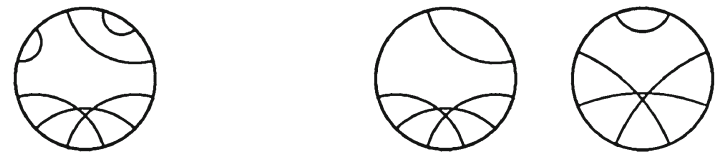

When $\Gamma^{\prime}$ has at least one chord with step 1, it has a father-diagram. The transfer from a diagram $\Gamma$ to its father-diagram may be iterated until one gets a diagram without chord with step 1; the latter is called leader-diagram of $\Gamma$.

For example, the leader-diagram of the Gauss diagram showed below on the left is the Gauss diagram represented on the right:
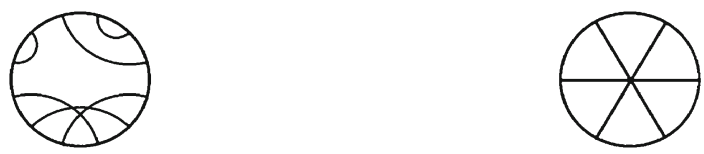

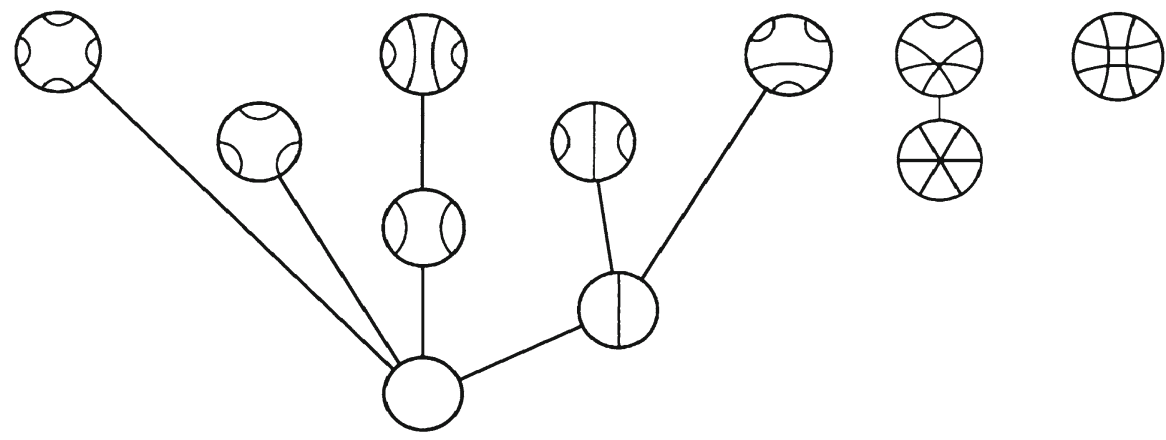

Fig. 4 The eleven Gauss diagrams of order $\leq 4$ belong to 3 families

Definition The family of a Gauss diagram $\Gamma$ is the (infinite) set of Gauss diagrams which have the same leader-diagram as $\Gamma$. The natural bijection between the set of the just defined families and the set of the leader-diagrams allows us to use the same drawing to denote a family and its leader-diagram.

The following properties, easy to prove, show that the concept family of diagrams is natural, especially for a classification of spherical curves.

1. The relation of fatherhood turns each family into a rooted tree. Three such trees are partially represented in Fig. 4.

2. All Gauss diagrams of a family have interlacing graphs with the same number of edges. More precisely, the interlacing graph of a diagram $\Gamma$ is isomorphic to the union of a totally disconnected graph and the interlacing graph of the leaderdiagram of the family of $\Gamma$.

3. If a Gauss diagram is realizable, then all the Gauss diagrams of its family are also realizable; hence one can say, in this case, that the family is realizable.

The way of constructing the Gauss diagrams of order $n>4$ combines two methods: the first one gives the list $N L(n)$ of the Gauss diagrams which are not leader-diagrams and is based on the catalogue of all Gauss diagrams whose orders are smaller than $n$; the second one gives the catalogue $L(n)$ of the leader-diagrams, and uses a part of the list $N L(n)$. We describe the first method immediately and delay the second one to Sect. 3 .

If $\Gamma$ belongs to $N L(n)$, it has a father-diagram which belongs to the catalogue of all Gauss diagrams whose orders are $<n$. Hence one will obtain the elements of $N L(n)$ by answering, for every Gauss diagram $\Gamma^{\prime}$ of order $<n$, the question:

what are the Gauss diagrams of order $n$ that have $\Gamma^{\prime}$ as father-diagram?

Let us consider the case where $n=5$ and $\Gamma^{\prime}$ is the Gauss diagram of order 3 represented hereunder; we must augment $\Gamma^{\prime}$ by means of two chords with step 1 .

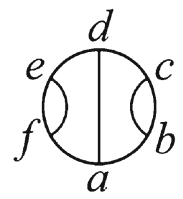


Let us try to put a first chord between $b$ and $c$, and a second one between $d$ and $e$. The result will be a diagram of order 5 whose father-diagram is the unique diagram of order 2 and not $\Gamma^{\prime}$.

To avoid this contradiction, it is necessary that the chords with step 1 in $\Gamma^{\prime}$ do not remain chords with step 1 in the new diagram; with other words:

At least one "new" chord with step 1 must be put in each edge of $\Gamma^{\prime}$ joining the endpoints of an "old" chord with step 1.

Obeying this rule and using the symmetries of $\Gamma^{\prime}$, one gets the complete list $N L(n)$ without a double occurrence.

\section{Gauss Diagrams of Order 5}

In the rest of this text, we use the abbreviation $k$-chord for chord with step equal to $k$. By adding 1-chords to Gauss diagrams of orders $<5$, as explained above, one gets the list $N L(5)$ that appears in Table 3.

The partial representation of the rooted trees drawn in Fig. 4 may now be extended to order 5; Fig. 5 shows the result for the second and the third families.

We now explain a method that may be applied to construct all leader-diagrams of a given order $n(n>3)$; for clarity, we expose it for $n=5$.

Among the elements of $N L(5)$, there are three Gauss diagrams without 3-chord; we now label their nodes by the integers modulo 10, in such a way that the neighbours of the node $k$ are the nodes $k-1$ and $k+1$.

Table 3 The 13 Gauss diagrams of order 5 which are not leader-diagrams

\begin{tabular}{|c|c|}
\hline $\begin{array}{c}\text { Father- } \\
\text { diagram }\end{array}$ & $\begin{array}{c}\text { Generated } \\
\text { Gauss diagrams }\end{array}$ \\
\hline 0 & \\
\hline 0
\end{tabular}

\begin{tabular}{|c|c|c|}
$\begin{array}{c}\text { Father- } \\
\text { diagram }\end{array}$ & Generated \\
Gauss diagrams
\end{tabular}



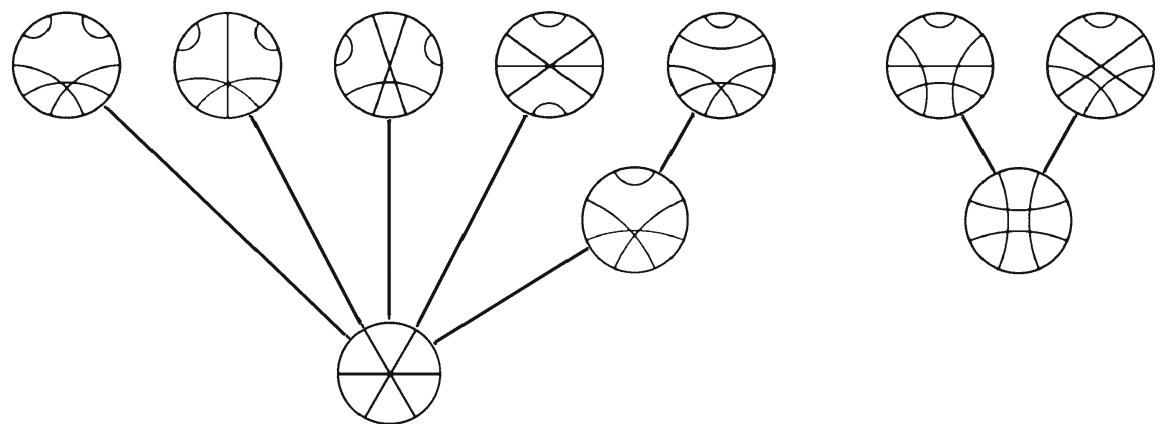

Fig. 5 Parts of the rooted trees starting with the leader-diagrams of order 3 and 4
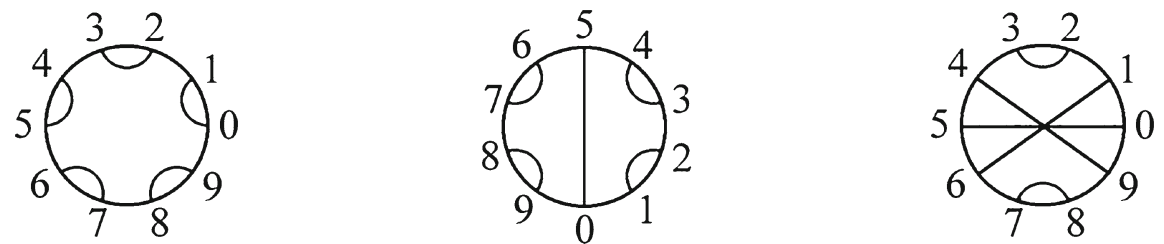

Let $\Gamma$ be any diagram above. As 3 is invertible in the ring $\mathbb{Z} / 10 \mathbb{Z}$, the multiplication by 3 defines a permutation $m_{3}$ of the nodes of $\Gamma$; we now replace the chords of $\Gamma$ by their images under $m_{3}$; each 1 -chord becomes a 3-chord and each 5-chord remains a 5-chord. So this construction provides 3 elements of $L(5)$ :
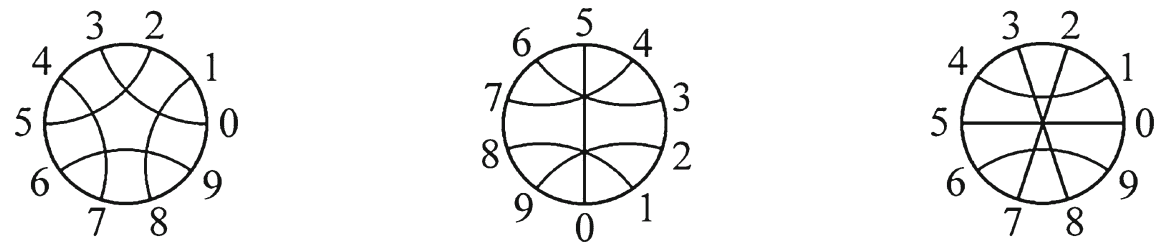

All Gauss diagrams with at least one 3-chord and without 1-chord are obtained by this procedure. In order to complete the list $L(5)$, it suffices to add the Gauss diagrams of order 5 which have neither 1-chord nor 3-chord; but this is easy: such a diagram is unique:

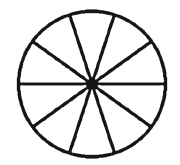

So there are 4 leader-diagrams of order 5.

\section{Gauss Diagrams of Order 6}

We proceed as for the Gauss diagrams of order 5, constructing first the elements of $N L(6)$; they are shown in the second column of Table 4. 
Table 4 The 44 Gauss diagrams of order 6 which are not leader-diagrams

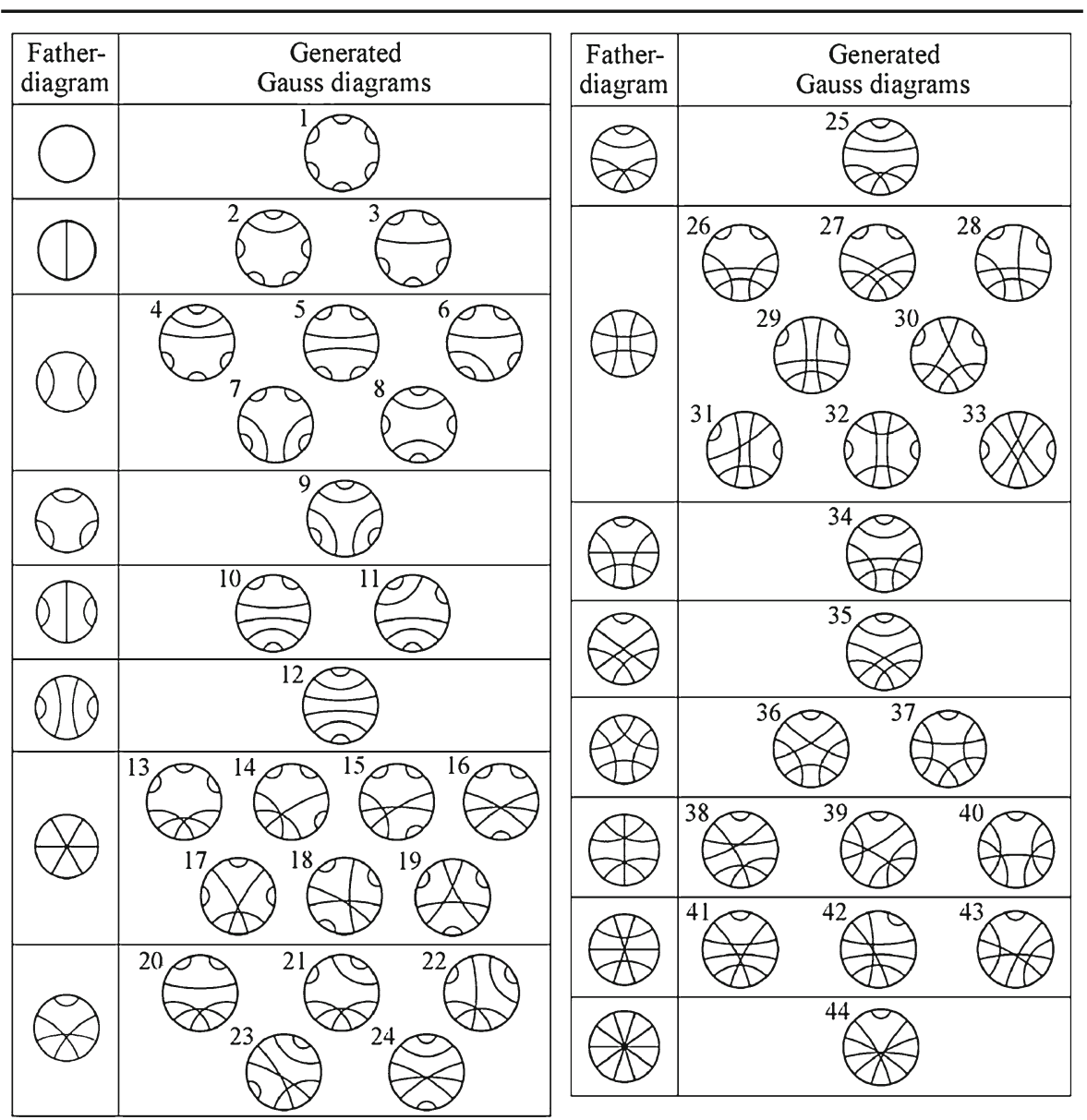

Among the 44 elements of $N L(6)$, seven diagrams have no 5-chords; they bear the numbers $1,2,7,8,9,13$ and 21 . If we label their nodes with the elements of the ring $\mathbb{Z} / 12 \mathbb{Z}$, and proceed as we did for the leader-diagrams of order 5 (now using the multiplication $m_{5}$ instead of $m_{3}$ ), we obtain 7 elements of $L(6)$, namely
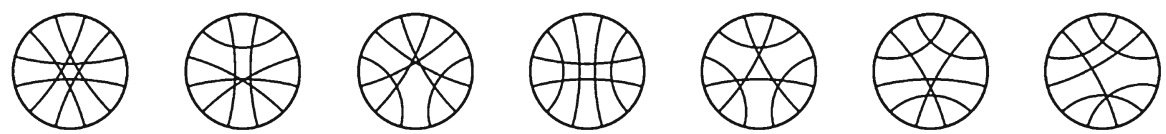

The elements of $L(6)$ which are not obtained in this way are the Gauss diagrams whose the step of every chord is 3 ; they are showed below (it is an easy exercise to see that there is no other). 

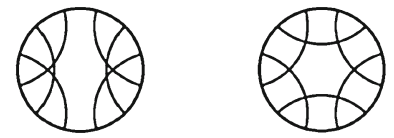

In short, there are 9 leader-diagrams of order 6.

\section{Gauss Diagrams of Order 7}

We have applied the procedure described at the end of Sect. 2 for drawing up the list of the elements of $N L(7)$; as it is a set of cardinality 217, we shall not annoy the reader with its description. However, for the reader who would like to check this cardinality, we decompose it as follows: the families

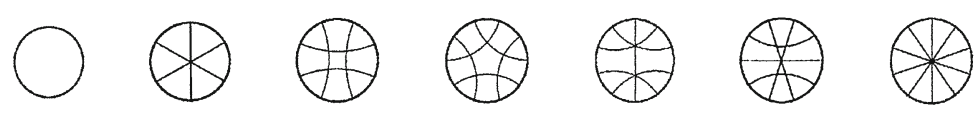

respectively include $27,48,34,11,21,21,7$ elements of $N L(7)$ and the union of the families generated by leader-diagrams of order 6 includes 48 elements of $N L(7)$.

The set $N L(7)$ contains 37 elements which have no 5-chord; these may be transformed (using the function $m_{3}$ in the ring $\mathbb{Z} / 14 \mathbb{Z}$ ) into diagrams without 1 -chord, but having at least one 3 -chord. The result gives a first part of $L(7)$ :
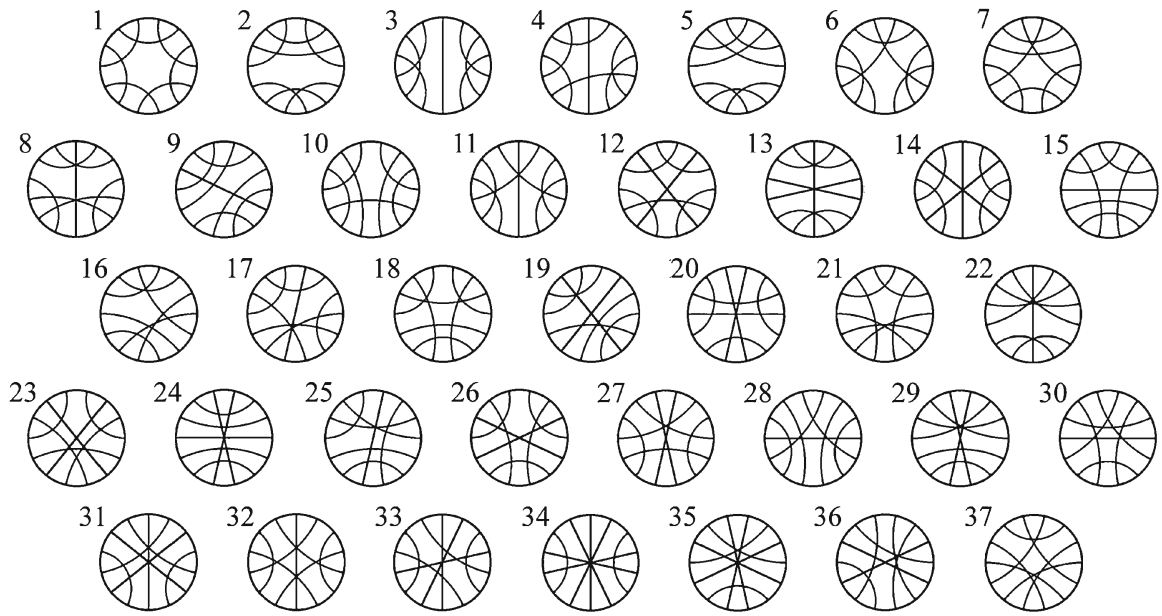

The other elements of $L(7)$ have neither 3-chord, nor 1-chord; if they have at least one 5-chord, they may be obtained (using the multiplication $m_{5}$ ) from the elements of $N L(7)$ having neither 5-chord, nor 3-chord. The result gives a second part of the set $L(7)$ :
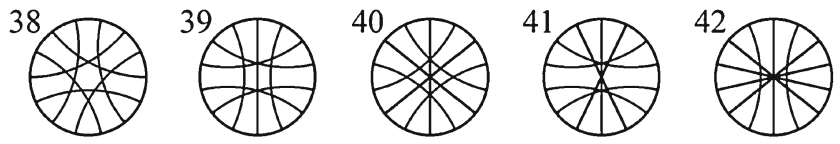
The last element of $L(7)$ is the diagram of order 7 which has only 7-chords.

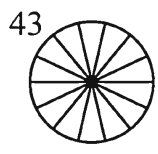

\section{The biparity condition}

The parity condition imposed in our definition of a Gauss diagram $\Gamma$ of order $n$ is not sufficient to insure that $\Gamma$ is realizable, i.e. is the Gauss diagram of a spherical curve. A second necessary condition of realizability is the

Biparity condition: If the chords $a a^{\prime}$ and $b b^{\prime}$ of $\Gamma$ are not interlaced, then the number of chords which are interlaced with aa' and with bb' is even.

Here is an example of a diagram that does not verify the biparity condition:

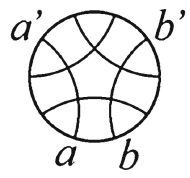

One notices that, if a Gauss diagram $\Gamma$ satisfies the biparity condition, then all the diagrams in the family of $\Gamma$ also satisfy it. In this case, one can say that the family of $\Gamma$ satisfies the biparity condition.

Among the families the leader of which has an order smaller than 5 (drawn hereafter), the biparity condition is always verified.
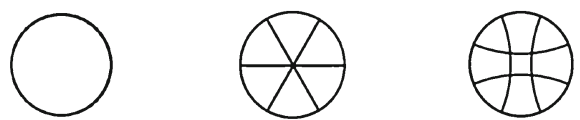

Among the families whose leader has order 5, the biparity condition is verified for half of the case, namely for the families
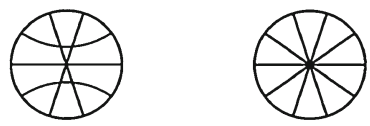

Among the families whose leader has order 6, the biparity condition is verified for 5 cases out of 9 ; here are the leaders of the 5 families
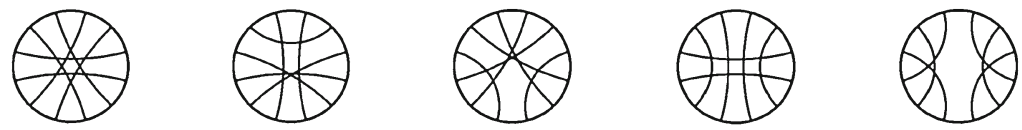

Among the families whose leader has order 7, the biparity condition is satisfied only in 14 instances out of 43; they are represented hereafter: 

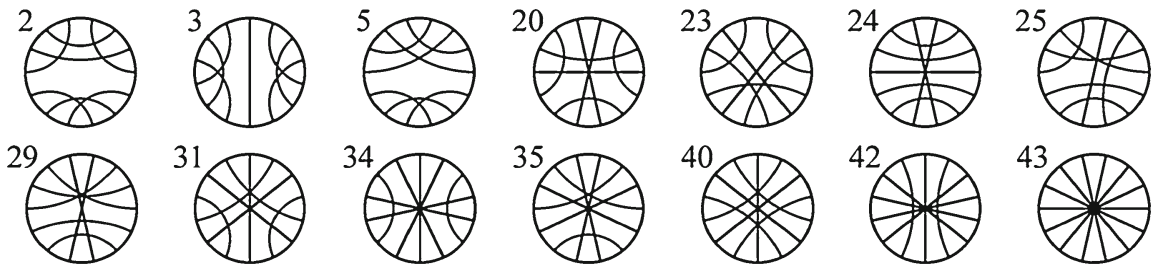

It remains to answer the questions, for every diagram among the 24 above: is it a realizable Gauss diagram? In the affirmative, what are the realizations?

\section{Spherical Realizations of Leader-Diagrams}

As it happens, most of the 24 leader-diagrams described in the preceding section are realizable. The exceptions are the diagram $\Gamma_{6}$ of order 6 whose every chord has step 5 and the diagram of order 7 numbered as 35 .

We prove by contradiction that $\Gamma_{6}$ is not realizable: suppose to the contrary that it is; then a vertex split at $a$ (see definition and property in Lovasz and Marx (1976)) would transform it into a realizable diagram $\Gamma_{5}$ :
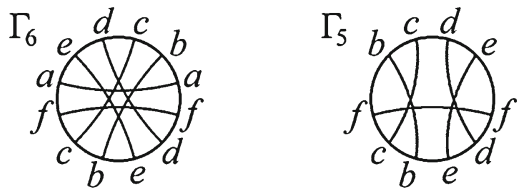

The contradiction is that $\Gamma_{5}$ does not verify the biparity condition, so it cannot be realizable.

An analogous reasoning proves that also the diagram 35 is not realizable.

\section{Families generated by a realizable leader-diagram of order $<8$.}

In Table 5, we associate a capital letter with each of the 22 families generated by a realizable leader-diagram of order $<8$.

One says that a Gauss diagram $\Gamma$ is decomposable if there exists a partition $\{A, B\}$ of the set of chords of $\Gamma$ such that no element of $A$ is interlaced with an element of $B$; this determines a decomposition of $\Gamma$ into two smaller diagrams; for example, the leader-diagram of family $\mathbf{V}$ has a decompostion consisting in the leader-diagrams of families $\mathbf{B}$ and $\mathbf{C}$.

Let us say that a Gauss diagram has essentially one realization if it is realizable and all its realizations belong to the same $\operatorname{Diff}\left(S^{2}\right)$-orbit. Using the informations given by any of the 22 realizable leader-diagrams of order not greater than 7 , one comes to the following conclusions:

(a) every leader-diagram of order $<6$ has essentially one realization,

(b) one leader-diagram of order 6 has two orbits of realizations, the other three just one,

(c) all but two leader-diagrams of order 7 have essentially one realization, one exception with two realizations and the other exception with three. 
Table 5 Names of the families including a realizable diagram of order $<8$

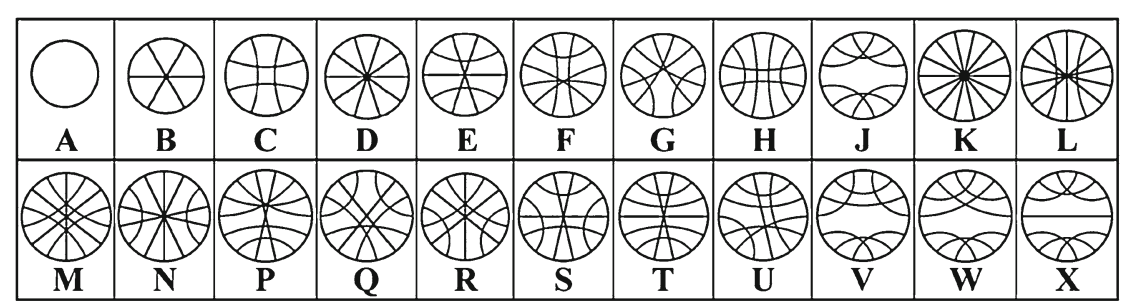

Pictures of curves with $n$ double points $(n<8)$ without any simple loop (realizations of leader-diagrams of order $<8$ ).

In Figs. 6 and 7, we exhibit drawings of realizations in increasing values of the order $n$ and, in case of equality of order, in decreasing values of the number of edges int of its interlacing graph. Sometimes, we show two plane representations which are $\operatorname{Diff}\left(S^{2}\right)$-equivalent; but in most cases, the chosen drawing is a curve with a maximum number of symmetries.

Remark (1) If $n \in\{2,3, \ldots, 7\}$, the leader-diagrams of order $n$ which are not decomposable are exactly the prime diagrams of order $n$ in the paper of Chmutov et al. (2006).

(2) The groups of diffeomeophisms preserving the realizations of the leader-diagrams $\mathbf{B}, \mathbf{C}, \mathbf{D}$ and $\mathbf{K}$ act transitively on their double points; the description of all spherical curves having this property is given in our paper (2016).
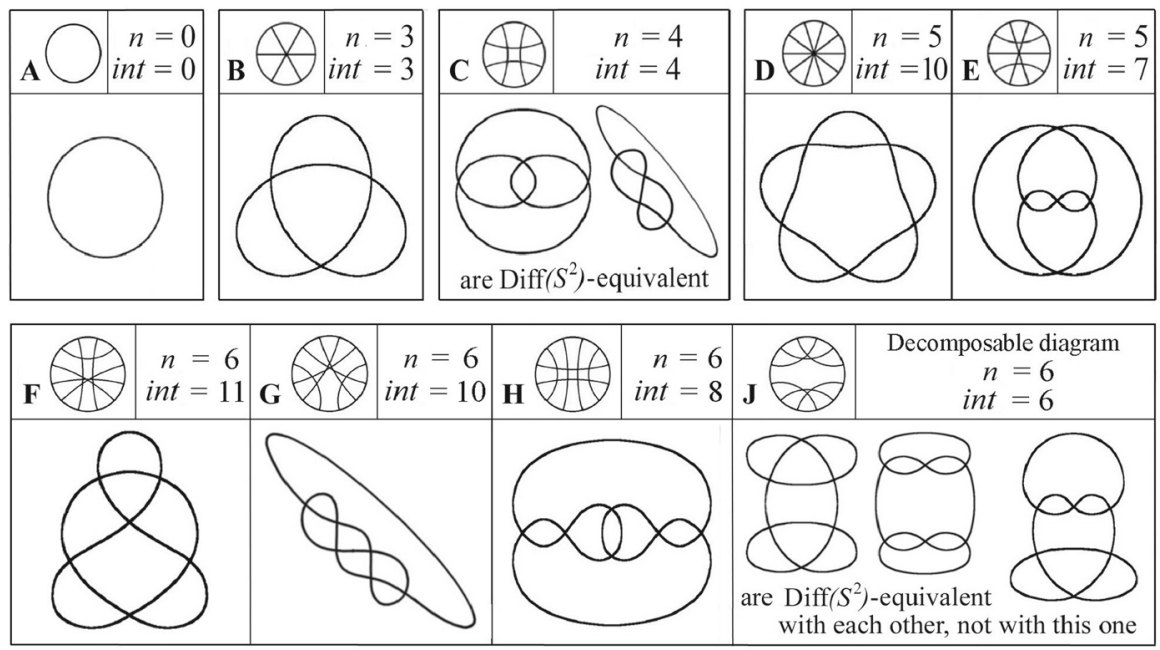

Fig. 6 Spherical realizations of the leader-diagrams of order $<7$ 

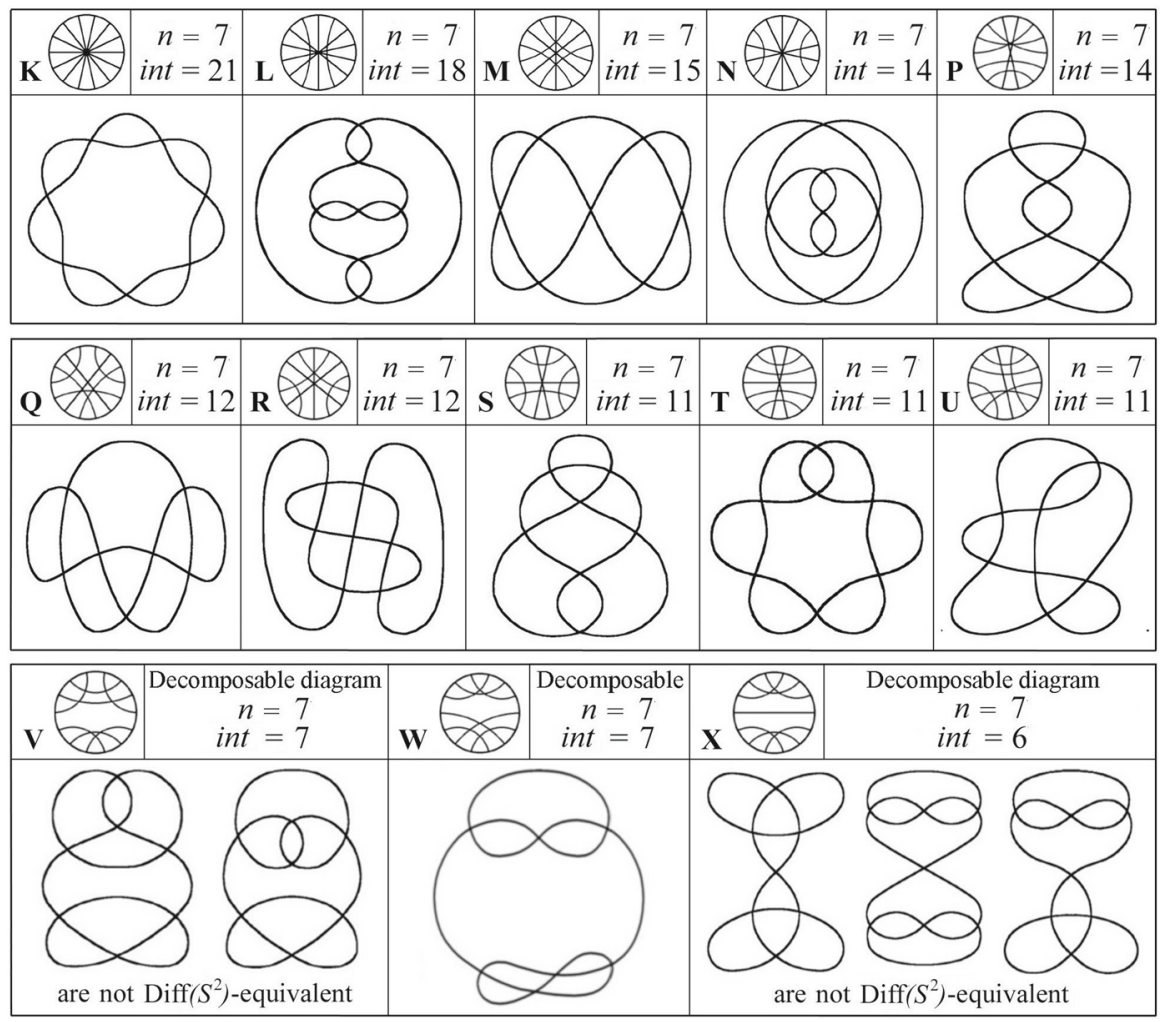

Fig. 7 Spherical realizations of the leader-diagrams of order 7

\section{Spherical Realizations of Other Gauss Diagrams}

The spherical realizations of a Gauss diagram $\Gamma$ which has a father-diagram could be done like those of the leader-diagrams, but this method is lengthy. We prefer to make use of the realizations of the father-diagram of $\Gamma$, which are supposed already known. The idea is simple: in each $\operatorname{Diff}\left(S^{2}\right)$-orbit of realizations of the father-diagram of $\Gamma$ we choose a model $M$, called mother-curve in the sequel, and we proceed as follows: for each edge of the father-diagram where some 1-chords were added to get $\Gamma$, we mark, by means of a short stroke, the corresponding open arc of $M$ which must be modify to give birth to a loop of the realization of $\Gamma$ in progress; an analysis of the combinatorial symmetries of $M$ enables to eliminate duplications of the resulting curve. We give additional explanations on this analysis by treating an example.

We first introduce some terminology: if $M$ is a spherical curve with at least one double point, we define an $M$-domain $D$ as a connected component of the complement of $M$ in $S^{2}$ and a side of $D$ as the closure of an open arc of the boundary of $D$ without double point and maximal for this property.

Imagine that we are looking for the spherical realizations of the diagram $\Gamma$ showed below on the left. The father-diagram of $\Gamma$, showed on the right, 

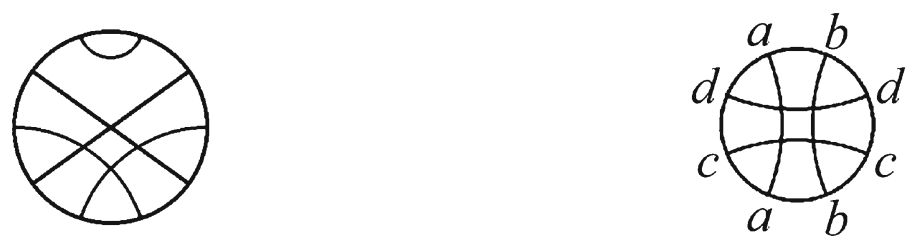

may be realized by several $\operatorname{Diff}\left(S^{2}\right)$-equivalent curves, for instance
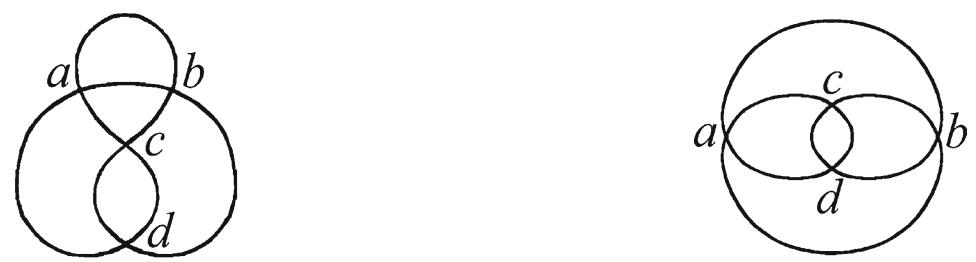

(we denote a point of $S^{1}$ and its image through a parametrization by the same letter). If $M$ is one of these models, the stabilizer $H$ of $M$ in the group $\operatorname{Diff}\left(S^{2}\right)$ acts transitively on the set of sides $a c, a d, b c$ and $b d$, so that we may choose $a d$ as arc where a loop will be added. Moreover, the model below, on the left, shows that there exists in $H$ a diffeomorphism permuting the $M$-domains $a d c$ and $d a b$.
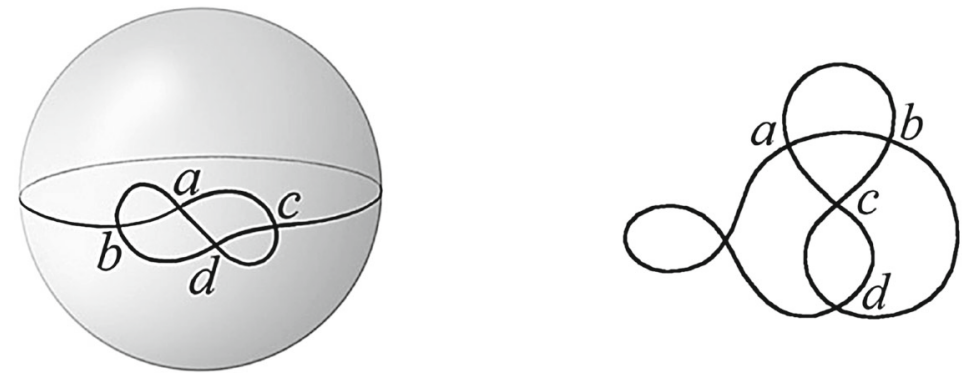

We may put the loop in $d a b$. Therefore there is essentially one spherical realization of the Gauss diagram $\Gamma$. It looks like the curve sketched on the right.

The method explained above allows to determine, for any Gauss diagram $\Gamma$, a complete set of spherical curves realizing $\Gamma$ (such a set $S$ is complete if any spherical curve with Gauss diagram $\Gamma$ is $\operatorname{Diff}\left(S^{2}\right)$-equivalent with exactly one element of $S$ ). Table 6 gives, for every family whose leader-diagram has order $<7$ and for every $n \leq 7$, the number of curves with $n$ double points, up to $\operatorname{Diff}\left(S^{2}\right)$-equivalence, which realize a Gauss diagram belonging to the family.

The Appendix concerning Table 6 shed light on the way to obtain the given values: beside every non leader-diagram $\Gamma$ appears the number of orbits of curves realizing $\Gamma$.

As a consequence, we obtain a classification of the spherical curves based on the number of double points and the absence of simple loops (Table 7). 
Table 6 Number of curves with $n$ double points, up to $\operatorname{Diff}\left(S^{2}\right)$-equivalence, which realize a Gauss diagram belonging to a given family

\begin{tabular}{rrrrrrrrrrr}
\hline$n$ & A & B & C & D & E & F & G & H & J & Totals \\
\hline 0 & 1 & & & & & & & & & 1 \\
1 & 1 & & & & & & & & & 1 \\
2 & 2 & & & & & & & & & 2 \\
3 & 5 & 1 & & & & & & & & 6 \\
4 & 16 & 2 & 1 & & & & & & & 19 \\
5 & 55 & 16 & 3 & 1 & 1 & & & & & 76 \\
6 & 240 & 92 & 31 & 2 & 6 & 1 & 1 & 1 & 2 & 376 \\
7 & 1149 & 654 & 228 & 22 & 76 & 14 & 12 & 8 & 15 & 2178 \\
\hline
\end{tabular}

Table 7 Summary without the families of diagrams

\begin{tabular}{lccc}
\hline \multirow{2}{*}{ Number of double points } & \multicolumn{2}{l}{ Number of orbits of spherical curves } & Total number of orbits \\
\cline { 2 - 3 } & Without simple loop & With simple loop or loops & \\
\hline 0 & 1 & 0 & 1 \\
1 & 0 & 1 & 1 \\
2 & 0 & 2 & 2 \\
3 & 1 & 5 & 6 \\
4 & 1 & 18 & 19 \\
5 & 2 & 74 & 76 \\
6 & 5 & 371 & 376 \\
7 & 16 & 2178 & 2194 \\
\hline
\end{tabular}

\section{Arnold Types}

Arnold (1994, 1996) distinguishes between five types of symmetry for plane curves; this classification is used by Gusein-Zade and Duzhin (1998) for determining the numbers of curves (oriented or not) with $n$ double points $(n \leq 10)$ in the plane (oriented or not).

We shall give an analogous classification of spherical curves, using following notations:

(a) If $C$ is a curve, we denote by $C^{+}$and $C^{-}$the oriented curves along $C$;

(b) We denote by $[C]$ the orbit of $C$ under $\operatorname{Diff}^{+}\left(S^{2}\right)$; we define analogously $\left[C^{+}\right]$ and $\left[C^{-}\right]$

(c) We denote by $-C$ the image of $C$ under the antipody of $S^{2}$, that is the restriction to $S^{2}$ of the operator $\mathbf{x} \rightarrow-\mathbf{x}$ in $\mathbb{R}^{3}$; we define analogously the oriented curves $-C^{+}$and $-C^{-}$. The orbit $\left[-C^{+}\right]$of $-C^{+}$under $\operatorname{Diff}^{+}\left(S^{2}\right)$ is also the set of the images of $C^{+}$under the elements of $\operatorname{Diff}\left(S^{2}\right) \backslash \operatorname{Diff}{ }^{+}\left(S^{2}\right)$; hence the equality $\left[f\left(C^{+}\right)\right]=\left[-C^{+}\right]$is valid for every oriented curve $C^{+}$and every reflection $f$. 
It is fairly obvious that the orbit of $C$ (resp. $C^{+}$, resp. $C^{-}$) under $\operatorname{Diff}\left(S^{2}\right)$ is $[C] \cup[-C]$ (resp. $\left[C^{+}\right] \cup\left[-C^{+}\right]$, resp. $\left[C^{-}\right] \cup\left[-C^{-}\right]$).

We now define the Arnold type of a spherical curve $C$ by looking at the possible coincidences of the orbits $\left[C^{+}\right],\left[C^{-}\right],\left[-C^{+}\right],\left[-C^{-}\right]$. Because each equality of two among these orbits implies the equality of the other two, the number of cases reduces to 5 .

(1) If the orbits $\left[C^{+}\right],\left[C^{-}\right],\left[-C^{+}\right],\left[-C^{-}\right]$are different from each other, then we say that the curve $C$ is asymmetric or of type Asy; this implies that the oriented curves $C^{+}$and $C^{-}$are not equivalent on the unoriented sphere and that the curves $C$ and $-C$ are not equivalent on the oriented sphere; in other words, the contribution of such a curve

to the number of orbits of oriented curves on the unoriented sphere is 2 , to the number of orbits of unoriented curves on the oriented sphere is 2 , to the number of orbits of oriented curves on the oriented sphere is 4 .

A 4-tuple summarizes these properties: $(1,2,2,4)$, later used as a column of a matrix.

Three examples of curves of type Asy are given hereafter.
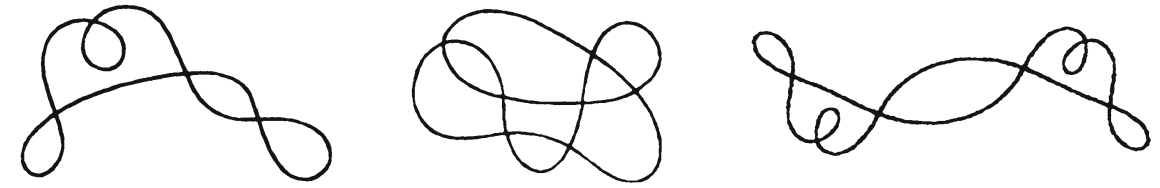

Remark that the last one is invariant under a half-turn and nevertheless is of type Asy.

(2) If the orbits $\left[C^{+}\right],\left[C^{-}\right],\left[-C^{+}\right]$and $\left[-C^{-}\right]$coincide, then we say that $C$ is supersymmetric or of type Sup; this implies that the oriented curves $C^{+}$and $C^{-}$are equivalent on the unoriented sphere and that the curves $C$ and $-C$ are equivalent on the oriented sphere; in other words, the contribution of $C$ to the number of orbits of oriented curves on the unoriented sphere is 1 , to the number of orbits of unoriented curves on the oriented sphere is 1 , to the number of orbits of oriented curves on the oriented sphere is 1 .

The 4-tuple related to the type Sup is thus $(1,1,1,1)$.

Four examples of curves of type Sup are given herafter.
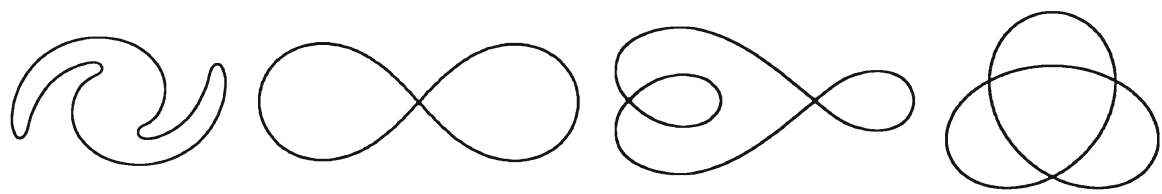

One easily sees that $\left[-C^{+}\right]=\left[C^{-}\right]$for the last two curves above; in order to be convinced that the equalities $\left[-C^{+}\right]=\left[C^{+}\right]$and $\left[C^{-}\right]=\left[C^{+}\right]$are also true for these curves, it is convenient to look at the following models: 

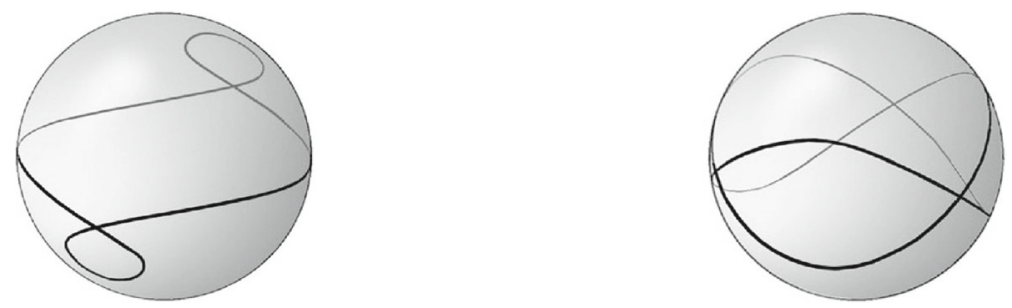

(3) If $\left[-C^{+}\right]=\left[C^{-}\right] \neq\left[-C^{-}\right]=\left[C^{+}\right]$, then we say that $C$ is symmetric(1) or of type $S y 1$; this implies that the oriented curves $C^{+}$and $C^{-}$are equivalent on the unoriented sphere and that the curves $C$ and $-C$ are equivalent on the oriented sphere; with other words, the contribution of such a curve

to the number of orbits of oriented curves on the unoriented sphere is 1 , to the number of orbits of unoriented curves on the oriented sphere is 1 , to the number of orbits of oriented curves on the oriented sphere is 2 .

The 4-tuple related to the type $S y l$ is thus $(1,1,1,2)$.

For instance, the curves hereunder are plane representations of spherical curves of type $S y 1$.
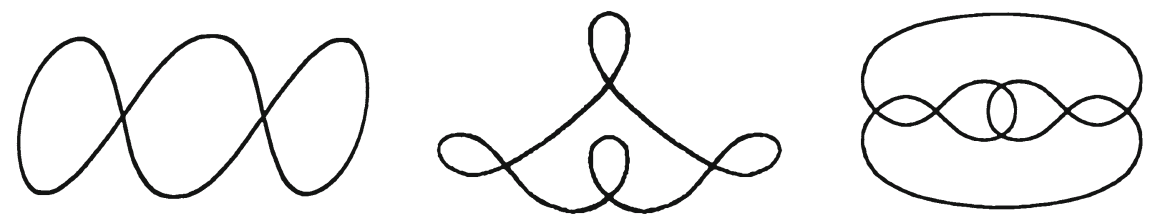

(4) If $\left[C^{-}\right]=\left[C^{+}\right] \neq\left[-C^{-}\right]=\left[-C^{+}\right]$, then we say that $C$ is symmetric(2) or of type $S y 2$; this implies that the oriented curves $C^{+}$and $C^{-}$are equivalent on the unoriented sphere and that the curves $C$ and $-C$ are not equivalent on the oriented sphere; with other words, the contribution of such a curve to the number of orbits of oriented curves on the unoriented sphere is 1 , to the number of orbits of unoriented curves on the oriented sphere is 2 , to the number of orbits of oriented curves on the oriented sphere is 2 .

The 4-tuple related to the type $S y 2$ is thus $(1,1,2,2)$.

The curves hereafter are models of spherical curves of type $S y 2$.
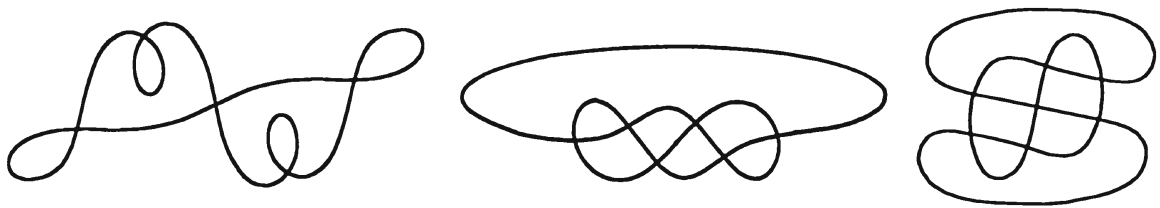

(5) If $\left[-C^{+}\right]=\left[C^{+}\right] \neq\left[-C^{-}\right]=\left[C^{-}\right]$, then we say that $C$ is symmetric(3) or of type $S y 3$; this implies that the oriented curves $C^{+}$and $C^{-}$are not equivalent on the unoriented sphere and that the curves $C$ and $-C$ are equivalent on the oriented sphere; with other words, the contribution of such a curve 
to the number of orbits of oriented curves on the unoriented sphere is 2 , to the number of orbits of unoriented curves on the oriented sphere is 1 , to the number of orbits of oriented curves on the oriented sphere is 2 . The 4-tuple related to the type $S y 3$ is thus $(1,2,1,2)$.

Examples of curves of type $S y 3$ are
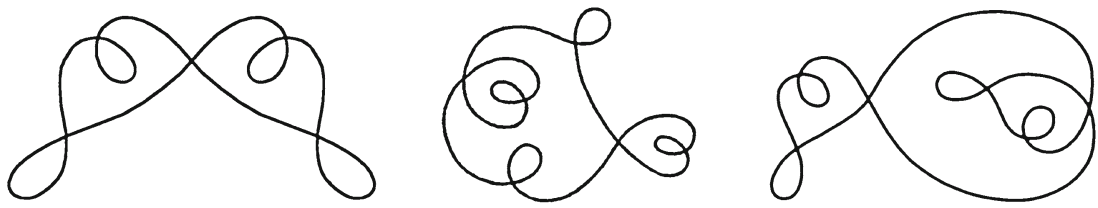

Because the symmetry type is the same for all curves in an orbit $K$ of $\operatorname{Diff}\left(S^{2}\right)$, one may speak of the symmetry type of $K$; we also say that $K$ realizes a diagram when the elements of $\mathrm{K}$ realize it.

If $\Gamma$ is a realizable diagram, then we associate with it the following integers:

$v$ (resp. $w, x, y, z$ ) is the number of orbits of type $\operatorname{Sup}($ resp. $S y 1, S y 2, S y 3, A s y$ ) which realize $\Gamma$;

$u u$ (resp. ou) is the number of $\operatorname{Diff}\left(S^{2}\right)$-orbits of unoriented (resp. oriented) curves which realize $\Gamma$ (the sphere is unoriented);

uo (resp. oo) is the number of $\operatorname{Diff}^{+}\left(S^{2}\right)$-orbits of unoriented (resp. oriented) curves which realize $\Gamma$ (the sphere is oriented).

N.B.: in the symbols $u u, \ldots, o o$, the first character is related to the curves and the second one to the sphere; $u$ is the initial of unoriented and $o$ begins oriented.

The stated properties of the five types of symmetry imply that $u u, o u, u o$ and $o o$ are linear functions of $v, w, x, y$ and $z$ :

$$
\begin{aligned}
& u и=v+w+x+y+z, \\
& \text { ои }=v+w+x+2 y+2 z, \\
& u o=v+w+2 x+y+2 z, \\
& \text { оо }=v+2 w+2 x+2 y+4 z .
\end{aligned}
$$

This system can be rewritten as a matrix equation

$$
\left[\begin{array}{c}
\text { uи } \\
\text { оu } \\
\text { ио } \\
\text { oо }
\end{array}\right]=\left[\begin{array}{lllll}
1 & 1 & 1 & 1 & 1 \\
1 & 1 & 1 & 2 & 2 \\
1 & 1 & 2 & 1 & 2 \\
1 & 2 & 2 & 2 & 4
\end{array}\right] \cdot\left[\begin{array}{l}
v \\
w \\
x \\
y \\
z
\end{array}\right]
$$

where the columns of the $4 \times 5$-matrix are the 4 -tuples related to the five symmetry types.

As an introduction to Sect. 10, suppose we want to determine the numbers $u и, o u, u o, o o$ of orbits which realize the Gauss diagram having one 5-chord and 
four 1-chords. The method explained in Sect. 7 produces seven orbits under $\operatorname{Diff}\left(S^{2}\right)$; they are represented by the curves
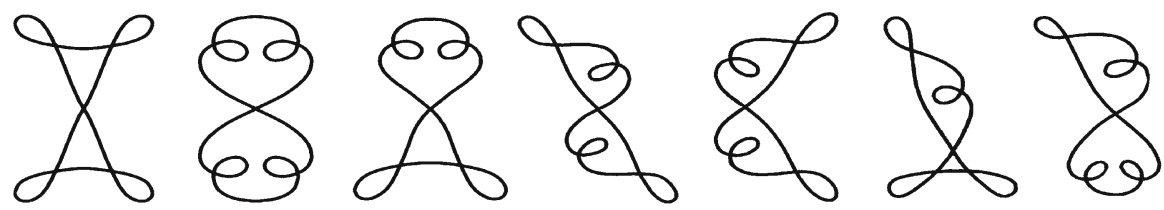

The first two curves have type Sup, the following three (from left to right), types $S y 1$, Sy2, Sy3, and the last two, type Asy; hence $v=2, w=x=y=1, z=2$ for the studied diagram and the matrix equation yields $u и=7, o u=10$, $и о=10$ and $o o=16$.

\section{Numerical Results}

\section{Counting the orbits of spherical curves with 5 double points}

Table 8 gets together the results of the enumeration of orbits of spherical curves with 5 double points; each row corresponds to a family, named in the first column; the second column gives the number of Gauss diagrams of order 5 in the family; the columns 3 to 7 give for each symmetry type $T$ (in the order $S u p, S y 1$, Sy2, Sy3, Asy), the number of orbits of type $T$ realizing diagrams of order 5 in the family; the columns 8 to 11 give

the number $U U$ of orbits of unoriented curves with 5 double points on the unoriented sphere,

the number $O U$ of orbits of oriented curves with 5 double points on the unoriented sphere,

the number $U O$ of orbits of unoriented curves with 5 double points on the oriented sphere,

the number $O O$ of orbits of oriented curves with 5 double points on the oriented sphere.

The last row gives total numbers; for example, the number of realizable Gauss diagrams of order 5 is 15 , the number of orbits of supersymmetric spherical curves with 5 double points is 10 (but there is only one orbit of type Sy3), the number of $\operatorname{Diff}^{+}\left(S^{2}\right)$-orbits of oriented curves is 182 .

As expected, the last four entries of the last row coincide with the values found by Arnold (see last column of first table in Sect. 1).

The Appendix concerning Table 8 sheds light on how to obtain the values given in it: beside every diagram $\Gamma$ in the considered family appear a 5-tupel giving the numbers $v, w, x, y, z$ defined at the end of Sect. 9, and the related 4-tuple giving the numbers $u u, o u, u o, o o$. So, the entry of this appendix describing the realizations of the diagram considered at the end of Sect. 9 is:

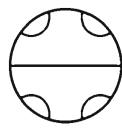

$(2,1,1,1,2)$

$(7,10,10,16)$ 
Table 8 Numbers of orbits of curves with 5 double points

\begin{tabular}{lcrrrrrrrrr}
\hline 1 & 2 & 3 & 4 & 5 & 6 & 7 & 8 & 9 & 10 & 11 \\
Family & \#GD & Su & S1 & S2 & S3 & As & UU & OU & UO & OO \\
\hline A & 6 & 6 & 31 & 1 & 1 & 16 & 55 & 72 & 72 & 136 \\
B & 5 & 2 & 9 & 2 & & 3 & 16 & 19 & 21 & 36 \\
C & 2 & & 2 & & & 1 & 3 & 4 & 4 & 8 \\
D & 1 & 1 & & & & & 1 & 1 & 1 & 1 \\
E & 1 & 1 & & & & & 1 & 1 & 1 & 1 \\
Total & 15 & 10 & 42 & 3 & 1 & 20 & 76 & 97 & 99 & 182 \\
\hline
\end{tabular}

Table 9 Numbers of orbits of curves with 6 double points

\begin{tabular}{lcrrrrrrrrr}
\hline 1 & 2 & 3 & 4 & 5 & 6 & 7 & 8 & 9 & 10 & 11 \\
Family & \#GD & Su & S1 & S2 & S3 & As & UU & OU & UO & OO \\
\hline A & 12 & 8 & 98 & 6 & 3 & 125 & 240 & 368 & 371 & 722 \\
B & 13 & & 36 & & & 56 & 92 & 148 & 148 & 296 \\
C & 10 & & 11 & 5 & & 15 & 31 & 46 & 51 & 92 \\
D & 1 & & 2 & & & & 2 & 2 & 2 & 4 \\
E & 3 & & 2 & & & 4 & 6 & 10 & 10 & 20 \\
F & 1 & & 1 & & & & 1 & 1 & 1 & 2 \\
G & 1 & & & 1 & & & 1 & 1 & 2 & 2 \\
H & 1 & & 1 & & & & 1 & 1 & 1 & 2 \\
J & 1 & 1 & 1 & & & & 2 & 2 & 2 & 3 \\
Total & 43 & 9 & 152 & 12 & 3 & 200 & 376 & 579 & 588 & 1143 \\
\hline
\end{tabular}

\section{Counting the orbits of spherical curves with 6 double points}

The number of realizable Gauss diagrams of order 6 is 43 . In the following table, we give, for each family, the numbers of orbits of unordered curves with 6 double points classified according to symmetry type, and the numbers of orbits if one supposes that the curves or the sphere are oriented.

The elements of the header of Table 9 have the same meaning as in Table 8 .

Remark (1) We got the last four values of the row "Total" of Table 9 in 2004 and added them to four sequences initiated by V. I. Arnold in the On-line Encyclopedia of Integer Sequences; the identifiers are A008989 for the $U U$-sequence, A008988, A008987 and AA008986 for the other three.

(2) The same remark, for $n=7$ instead of $n=6$, is valid for the last four values of the row "Totals" in Table 10. 
Table 10 Numbers of orbits of curves with 7 double points

\begin{tabular}{lrrrrrrrrrr}
\hline Family & \#GD & Su & S1 & S2 & S3 & \multicolumn{1}{c}{ As } & UU & OU & UO & OO \\
\hline A & 27 & 16 & 288 & 12 & 12 & 821 & 1149 & 1982 & 1982 & 3924 \\
B & 48 & 8 & 136 & 14 & 2 & 494 & 654 & 1150 & 1162 & 2288 \\
C & 34 & & 40 & & & 188 & 228 & 416 & 416 & 832 \\
D & 7 & 2 & 11 & 4 & & 5 & 22 & 27 & 31 & 52 \\
E & 21 & 2 & 11 & 4 & 4 & 55 & 76 & 135 & 135 & 260 \\
F & 7 & & 4 & & & 10 & 14 & 24 & 24 & 48 \\
G & 7 & & & & & 12 & 12 & 24 & 24 & 48 \\
H & 4 & & 4 & & & 4 & 8 & 12 & 12 & 24 \\
J & 4 & & 6 & & & 9 & 15 & 24 & 24 & 48 \\
K & 1 & 1 & & & & & 1 & 1 & 1 & 1 \\
L & 1 & 1 & & & & & 1 & 1 & 1 & 1 \\
M & 1 & 1 & & & & & 1 & 1 & 1 & 1 \\
N & 1 & 1 & & & & & 1 & 1 & 1 & 1 \\
P & 1 & & 1 & & & & 1 & 1 & 1 & 2 \\
Q & 1 & & 1 & & & & 1 & 1 & 1 & 2 \\
R & 1 & & & 1 & & & 1 & 1 & 2 & 2 \\
S & 1 & & 1 & & & & 1 & 1 & 1 & 2 \\
T & 1 & 1 & & & & & 1 & 1 & 1 & 1 \\
U & 1 & & & & & 1 & 1 & 2 & 2 & 4 \\
V & 1 & & 2 & & & & 2 & 2 & 2 & 4 \\
W & 1 & & & & & 1 & 1 & 2 & 2 & 4 \\
X & 1 & 2 & 1 & & & & 3 & 3 & 3 & 4 \\
Total & 172 & 35 & 506 & 35 & 18 & 1600 & 2194 & 3812 & 3829 & 7553 \\
\hline
\end{tabular}

(3) In a recent paper, Robert Coquereaux and Zuber (2016) use another method to count the last four numbers of the rows "Total" in Tables 8, 9 and 10 in their Table 5 (p. 25), they confirm our results without using our 22 families.

(4) The interested reader who wants to know about the contributions of a specific Gauss diagram of order 6 to the numbers $S u, S 1, \ldots, U O, O O$ (or comparing her/his own counts with ours) may consult the Appendix concerning Table 9.

\section{Counting the orbits of spherical curves with $\mathbf{7}$ double points}

The number of realizable Gauss diagrams of order 7 is 172 (13 are leader-diagrams and 159 are not). Table 10 is analogous to Tables 8 and 9, giving now informations about curves with 7 double points in each of the 22 considered family.

The contributions of a given Gauss diagram to the numbers $S u, S 1, \ldots, U O, O O$ are detailed in the Appendix concerning Table 10.

In Table 11, we give, for each symmetry type $\mathrm{T}$, the numbers of orbits of unordered curves of type $\mathrm{T}$ classified according to their number of double points. 
Table 11 Numbers of orbits of curves with a given symmetry type.

\begin{tabular}{lllllrrrr}
\hline Type & $n=0$ & 1 & 2 & 3 & \multicolumn{1}{c}{4} & \multicolumn{1}{c}{6} & \multicolumn{1}{c}{7} \\
\hline Sup & 1 & 1 & 1 & 3 & 5 & 10 & 9 & 35 \\
Sy1 & & & 1 & 3 & 12 & 42 & 152 & 506 \\
Sy2 & & & & & & 3 & 12 & 35 \\
Sy3 & & & & & & 1 & 3 & 18 \\
Asy & & & & & & 20 & 200 & 1600 \\
Total & 1 & 1 & 2 & 6 & 19 & 76 & 176 & 2194 \\
\hline
\end{tabular}

Table 12 Numbers of orbits of curves wthout simple loop and

\begin{tabular}{lllllllll}
\hline Oriented object(s) & $n=0$ & 1 & 2 & 3 & 4 & 5 & 6 & 7 \\
\hline Sphere and curves & 1 & 0 & 0 & 1 & 1 & 2 & 9 & 29 \\
Sphere & 1 & 0 & 0 & 1 & 1 & 2 & 6 & 19 \\
Curves & 1 & 0 & 0 & 1 & 1 & 2 & 5 & 18 \\
None & 1 & 0 & 0 & 1 & 1 & 2 & 5 & 16 \\
\hline
\end{tabular}

In a last table, we consider only curves without any simple loop (realizations of leader-diagrams); the numbers of orbits of such curves (oriented or not) under $\operatorname{Diff}\left(S^{2}\right)$ or $\operatorname{Diff}^{+}\left(S^{2}\right)$ ) are given in Table 12. The given values also appear in the Table 6 (p. 26) of Coquereaux and Zuber (2016).

Acknowledgments Grateful thanks are due to F. Aicardi, J.-P. Doignon, S. Duzhin and J.-B. Zuber who encouraged me to write and publish this work. I also thank the referee for helpful comments on the first version of the paper.

\section{References}

Arnold, V.I.: Topological invariants of plane curves and caustics, University lecture series. Am. Math. Soc. 5, 60 (1994)

Arnold, V.I.: Remarks on the enumeration of plane curves. Am. Math. Soc. Transl. (2) 173, 17-32 (1996)

Chaves, N., Weber, Cl: Plombages de rubans et problème des mots de Gauss. Expo. Math. 12, 53-77 (1994)

Chmutov, M., Hulse, T., Lum, A., Rowell P.: Plane and spherical curves: an investigation of their invariants.

Proceedings of the research experiences for undergraduates program in mathematics, Oregon State University (2006)

Coquereaux, R., Zuber, J.-B.: Maps, immersions and permutations. J. Knot Theory Ramif. (2016, to appear) Dowker, C.H., Thistlethwaite, M.B.: Classifications of knot projections. Topol. Appl. 16, 19-31 (1983)

Francis, G.K.: Null genus relizability criterion for abstract intersection sequences. J. Comb. Th. 7, 331-341 (1969)

Gauss, C.F.: Zur Geometria Situs, Werke (posthumous), vol. 8, pp. 271-286 (1900)

Gusein-Zade, S.M., Duzhin, F.S., On the number of topological types of plane curves. Usp. Mat. Nauk 53(3)(321), 197-198 (1998)

Lovasz, L., Marx, M.L.: A forbidden substrucure characterization of Gauss codes. Acta. Sci. Math. Soc. 38, 115-119 (1976)

Marx, M.L.: The Gauss realizability problem. Proc. Amer. Math. Soc. 22, 610-613 (1969)

Rosenstiehl, P., Read, R.C.: On the Gauss crossing problem. Proc. Fifth Hung. Comb. Colloq. pp. 843-876 (1977)

Valette, G.: Double point-homogeneous spherical curves. Bull. Belg. Math. Soc. Simon Stevin 23, 73-86 (2016) 